\title{
Publisher's Note: Cluster dynamical mean field theory study of antiferromagnetic transition in the square-lattice Hubbard model: Optical conductivity and electronic structure
} [Phys. Rev. B 94, 085110 (2016)]

Toshihiro Sato and Hirokazu Tsunetsugu

(Received 12 August 2016; published 22 August 2016)

DOI: 10.1103/PhysRevB.94.079907

This paper was published online on 8 August 2016 with an error in the Acknowledgments. The last sentence of the Acknowledgments should read as "This work has been supported by a Grant-in-Aid for Scientific Research from the Japan Society for the Promotion of Science (Grant No. 25400359)." The Acknowledgments have been corrected as of 12 August 2016. The Acknowledgments are correct in the printed version of the journal. 